\title{
Prognostic value of glomerular filtration rate and proteinuria in patients with diabetes mellitus and advanced chronic kidney disease
}

\author{
Tomoyuki Kawada ${ }^{1}$ (D)
}

Received: 12 August 2017/ Accepted: 15 August 2017/Published online: 22 August 2017

(C) Japanese Society of Nephrology 2017

\section{To the Editor,}

Chen et al. evaluated the prognosis of 447 diabetic patients with advanced chronic kidney disease (CKD), aged from 18 to 80 [1]. By Cox regression analysis, adjusted hazard ratios of patients with $\geq$ Stage 4 and severe proteinuria for renal replacement therapy (RRT) or mortality significantly increased. In addition, adjusted hazard ratios of estimated glomerular filtration rate for RRT (or mortality) significantly increased with dose-response manner. I have some concerns about their study.

First, the authors recognized 140 patients with RRT, 36 patients expired and 65 patients had cardiovascular events. Although there were no significant hazard ratios for mortality or cardiovascular events, there is a possibility of insufficient number of events. The number of events per independent variable in Cox regression analysis should be 10 or higher to keep statistical power [2,3]. In cases of mortality and cardiovascular event, the number of independent variables should be under 4 and 7 , respectively. Continuous survey is needed to keep enough number of events.

Relating to the first query, the authors used the modified diabetic nephropathy score by considering the excretion of urinary albumin [4], but a significant dose-response relationship between modified diabetic nephropathy scores and RRT (or mortality) showed unstable estimate with a wide range of $95 \%$ confidence intervals.

Finally, the authors used data of patients with wide ranges of age. Hazard ratio of patients aged 60 or older for

Tomoyuki Kawada

kawada@nms.ac.jp

1 Department of Hygiene and Public Health, Nippon Medical School, 1-1-5 Sendagi, Bunkyo-Ku, Tokyo 113-8602, Japan
RRT significantly decreased. The reason of protective effect by aging for RRT should be specified by further study.

\section{Compliance with ethical standards}

Conflict of interest The author declares no conflict of interest and no financial support.

Informed consent There is no need of informed consent in this study.

\section{References}

1. Chen PM, Wada T, Chiang CK. Prognostic value of proteinuria and glomerular filtration rate on Taiwanese patients with diabetes mellitus and advanced chronic kidney disease: a single center experience. Clin Exp Nephrol. 2017;21(2):307-15. doi:10.1007/ s10157-016-1290-8.

2. Concato J, Peduzzi P, Holford TR, Feinstein AR. Importance of events per independent variable in proportional hazards analysis. I. Background, goals, and general strategy. J Clin Epidemiol. 1995;48(12):1495-501. doi:10.1016/0895-4356(95)00510-2.

3. Peduzzi P, Concato J, Feinstein AR, Holford TR. Importance of events per independent variable in proportional hazards regression analysis. II. Accuracy and precision of regression estimates. J Clin Epidemiol. 1995;48(12):1503-10. doi:10.1016/0895-4356(95) 00048-8.

4. Levey AS, Eckardt KU, Tsukamoto Y, Levin A, Coresh J, Rossert $\mathrm{J}$, et al. Definition and classification of chronic kidney disease: a position statement from kidney disease: improving global outcomes (KDIGO). Kidney Int. 2005;67(6):2089-100. doi:10.1111/j. 1523-1755.2005.00365.x. 